\title{
Isolamento e identificação da microbiota periodontal de cães da raça Pastor Alemão
}

\author{
Isolate and identify of periodontal microbiota of German Shepherd dogs
}

\author{
Carla Afonso da Silva Bitencourt Braga ${ }^{1}$ Cleusa Maria Faria Resende ${ }^{2}$ \\ Ana Cristina Nogueira Rodrigues Pestana ${ }^{3}$ Luiz Semeão Carmo ${ }^{4}$ José Eustáquio Costa ${ }^{5}$ \\ Luiz Antonio Franco Silva ${ }^{6}$ Lílian Nery de Assis ${ }^{7}$ Larissa de Assis Lima ${ }^{7}$ \\ Luiz Macedo Farias ${ }^{8}$ Maria Auxiliadora Roque Carvalho ${ }^{8}$
}

\section{RESUMO}

A microbiota indígena gengival de cães não está totalmente descrita, sendo sua identificação uma etapa importante no estabelecimento da etiopatogenia e terapia da doença periodontal. O objetivo deste trabalho foi isolar $e$ identificar a microbiota periodontal de cães da raça Pastor Alemão, considerando sítios saudáveis e com doença periodontal. Foram utilizados 29 cães, com idade variando de três a seis anos, sendo analisados espécimes clínicos de sítios periodontais saudáveis de 12 cães e sítios com periodontite de outros 17. Foram isoladas 672 amostras microbianas, com predomínio dos gêneros Pasteurella, Staphylococcus, Porphyromonas e Fusobacterium. A microbiota dos sítios saudáveis equiparou-se à dos sítios doentes, tratando-se de uma microbiota indígena. A microbiota dos sítios doentes apresentou-se aumentada em relação a dos sítios saudáveis, indicando mudança do ambiente do sítio periodontal.

Palavras-chave: doença periodontal, cães, microbiota.

\section{ABSTRACT}

The indigenous gingival microbiota of dogs is not totally described, although such identification is an important step to establish the etiopathogenesis and adequate therapy for the periodontal disease. The aims of this study were to isolate and identify the periodontal microbiota of German Shepherd dogs from healthy and with periodontal desease sites. Twenty nine German Shepherd dogs from three to six years of age were used in this study. Clinical specimens were analysed from healthy periodontal sites of 12 dogs and sites presenting gingivitis of 17 dogs. A total amount of 672 microbial samples, were isolated where the predominant genera were Pasteurella spp., Staphylococcus spp., Porphyromonas spp. and Fusobacterium spp. The microbiological population of the affected sites was similar to the healthy sites, consisting on an indigenous microbiota. The microbiota on the affecteded sites was higher in number than on the healthy sites, showing change in the environment of the periodontal sites.

Key words: periodontal disease, dogs, microbiota.

\section{INTRODUÇÃO}

A microbiota indígena oral de humanos e animais é extensamente diversificada e complexa, necessitando de maiores estudos sobre sua composição e interação. Esses microrganismos estão freqüentemente envolvidos em muitas doenças infecciosas anaeróbias polimicrobianas como as doenças periodontais (FINEGOLD, 1995). Estas podem ser classificadas em gengivite, inflamação restrita ao tecido gengival livre, e periodontite, forma mais grave, na qual ocorre perda óssea e eventual perda do dente (HARVEY \& EMILY, 1993).

${ }^{1}$ Professor de Microbiologia, Doutor, Campus Avançado de Jataí da Universidade Federal de Goiás (UFG), Av. Dorival de Carvalho 996A, Centro, 75.800-014, Jataí-GO. E-mail: carlaafonso@bol.com.br

${ }^{2}$ Professor de Clínica Cirúrgica Animal, Doutor, Escola de Veterinária, Universidade Federal de Minas Gerais (UFMG).

${ }^{3}$ Aluno de Doutorado, Instituto de Ciências Biológicas, UFMG.

${ }^{4}$ Pesquisador, Doutor, Fundação Ezequiel Dias, Belo Horizonte, MG.

${ }^{5}$ Professor de Periodontia, Mestre, Escola de Odontologia, UFMG.

${ }^{6}$ Professor de Clínica Cirúrgica Animal, Doutor, Escola de Veterinária, UFG.

${ }^{7}$ Pesquisador, Biólogo Estagiário do Laboratório de Microbiologia, Campus Avançado de Jataí, UFG

${ }^{8}$ Pesquisador, Doutor, Instituto de Ciências Biológicas, UFMG. 
Recentes estudos têm enfatizado também a importância da microbiota oral de cães no desenvolvimento de infecções por meio de contaminação de feridas por mordidas em seres humanos e outros animais (GOLDSTEIN et al., 1984; GOLDSTEIN, 1998; TALAN et al., 1999), e em contaminações de feridas cirúrgicas de cães devido à interferência dos mesmos.

O objetivo deste trabalho foi isolar e identificar a microbiota de sítios periodontais saudáveis e com doença periodontal de cães da raça Pastor Alemão, sendo estes animais comumente utilizados como cães de guarda e em ações da Polícia Militar de Minas Gerais.

\section{MATERIAL E MÉTODOS}

Foram utilizados 29 cães da raça Pastor Alemão, 13 machos e 16 fêmeas, com peso médio de $29,1 \mathrm{~kg}$ e idade variando entre três e seis anos, provenientes do canil da Polícia Militar de Belo Horizonte, Minas Gerais. Os animais eram mantidos em canis individuais, recebendo água ad libitum e ração comercial ${ }^{a}$ extrudada com carne bovina crua e moída, uma vez ao dia.

A avaliação clínica do periodonto e colheita das amostras foram realizadas após administração de cloridrato de xilazina ${ }^{\mathrm{b}}$, na dose de $1,0 \mathrm{mg} \mathrm{kg}^{-1}$ de peso, por via intramuscular, tendo sido os animais posicionados em decúbito lateral esquerdo. Após o exame bucal, a colheita das amostras foi feita em sítios periodontais saudáveis de 12 cães (Grupo I) e em sítios com doença periodontal de 17 cães (Grupo II), utilizando-se pontas de papel absorvente introduzidas no interior dos sulcos gengivais. As pontas de papel foram imersas em solução de Ringer PRAS (Prereduced Anaerobically Sterilized), conforme as indicações de SUMMANEN et al. (1993), sob fluxo de gás carbônico $\left(\mathrm{CO}_{2}\right)$, transportadas para o laboratório e processadas em câmara anaeróbica ${ }^{\mathrm{c}}(5 \%$ $\mathrm{CO}_{2}, 10 \% \mathrm{H}_{2}$ e $85 \% \mathrm{~N}_{2}$ ). Em seguida foram empregados os meios de cultura TSAS ${ }^{\text {d }}$ (Tryptic Soy Agar) suplementado com hemina ${ }^{\mathrm{e}}\left(0,05 \mathrm{mg} \mathrm{ml}^{-1}\right)$, menadiona $^{\mathrm{f}}\left(0,01 \mathrm{mg} \mathrm{ml}^{-1}\right)$, extrato de levedura ${ }^{\mathrm{g}}(5 \mathrm{mg}$ $\mathrm{ml}^{-1}$ ) e acrescido de sangue desfibrinado de carneiro/ cavalo (5\%); TSBV (Tryptic Soy Serum Bacitracin Vancimicin), BBE (Ágar Bacteroides Bile Esculina); Ágar Omata \& Disraely; Ágar MacConkey e Ágar Sabouraud ${ }^{\mathrm{i}}$ acrescido de $100 \mu \mathrm{g} \mathrm{ml}^{-1}$ de cloranfenicol $^{\mathrm{j}}$ (SUMMANEN et al., 1993; KONEMAN et al., 2001). Os meios para isolamento de bactérias anaeróbias estritas (TSAS, TSBV, BBE, Omata \& Disraely) foram incubados a $37^{\circ} \mathrm{C}$, em câmara anaeróbica, realizando-se a leitura a partir de 48 horas. O TSAS suplementado foi mantido incubado em anaerobiose por até 14 dias, para o isolamento de bactérias produtoras de pigmento negro.

Os meios para isolamento de bactérias aeróbias, incluindo anaeróbios facultativos e microaerófilos (TSAS, MacConkey) foram incubados em aerobiose, a $37^{\circ} \mathrm{C}$, em estufa bacteriológica BOD, por um período de 48 horas. O meio de ágar Sabouraud foi mantido em temperatura ambiente, sendo as leituras realizadas a partir do quinto dia, por até 21 dias.

Após o período de incubação, passou-se à obtenção de culturas puras e identificação preliminar, segundo características morfocoloniais, morfotintoriais e teste respiratório (SUMMANEN et al., 1993). As leveduras obtidas foram conservadas em ágar Sabouraud, a $4^{\circ} \mathrm{C}$, e subcultivadas a cada três meses até a sua identificação definitiva. Cada grupo bacteriano foi identificado por características bioquímico-fisiológicas convencionais e por kits comerciais (SNEATH et al.,1986; BARON et al., 1994; HOLT et al., 1994; QUINN et al., 1994; SUMANNEN et al., 1993; KONEMAN et al., 2001) e, o grupo de leveduras, segundo BARON et al. (1994), GÜEHO et al. (1996), GUILLOT et al. (1996) e DUARTE et al. (1999).

A análise estatística foi feita segundo o teste exato de Fisher (JOHNSON \& BHATTACHARYYA, 1986) sendo considerado significativo para uma probabilidade de significância menor que $5 \%(\mathrm{P}<0,05)$ com, pelo menos $95 \%$ de confiança nas conclusões apresentadas.

\section{RESULTADOS E DISCUSSÃO}

Ao exame físico periodontal, foi observado que $27(93,10 \%)$ cães apresentavam vários sítios com quadro clínico de gengivite, com sangramento à sondagem e edema gengival e, somente dois $(6,90 \%)$, apresentavam todos os sítios avaliados saudáveis. As características clínicas para classificar as lesões observadas como gengivite estão de acordo com as descritas por HARVEY \& EMILY (1993).

Seiscentas e setenta e duas amostras microbianas foram isoladas dos 29 cães, sendo 379 $(56,40 \%)$ bactérias anaeróbias estritas, $236(35,12 \%)$ anaeróbias facultativas, $46(6,84 \%)$ aeróbias estritas ou microaerófilas e onze $(1,64 \%)$ leveduras. A identificação microbiana permitiu o agrupamento dos isolados em 49 espécies diferentes, discriminadas nas tabelas 1, 2 e 3. Grande parte deles corresponde aos gêneros microbianos mais relatados pela literatura (BOYCE et al., 1995; HARVEY et al., 1995; 
Tabela 1 - Distribuição das bactérias anaeróbias estritas isoladas de sítios periodontais saudáveis e com doença periodontal (DP) de 29 cães da raça Pastor Alemão (Minas Gerais, Brasil).

\begin{tabular}{|c|c|c|c|}
\hline \multirow{2}{*}{ Microbiota anaeróbia } & \multicolumn{2}{|c|}{ Distribuição das amostras } & \multirow{2}{*}{ Total } \\
\hline & Saudável & $\mathrm{DP}$ & \\
\hline $\begin{array}{l}\text { Fusobacterium } \\
\text { zucleatum }\end{array}$ & 18 & 55 & 73 \\
\hline Fusobacterium russi & 0 & 24 & 24 \\
\hline Bracteroides gracilis & 6 & 0 & 6 \\
\hline $\begin{array}{l}\text { Bacteroides } \\
\text { pneumosintes }\end{array}$ & 3 & 0 & 3 \\
\hline Bacteroides uniformis & 1 & 2 & 3 \\
\hline Bacteroides ovatus & 0 & 1 & 1 \\
\hline Bacteroides eggertii & 0 & 3 & 3 \\
\hline Bacteroides capillosus & 0 & 2 & 2 \\
\hline Porphyromonas spp. & 14 & 66 & 80 \\
\hline $\begin{array}{l}\text { Prevotella } \\
\text { intermédia/nigrescens }\end{array}$ & 0 & 18 & 18 \\
\hline $\begin{array}{l}\text { Prevotella ruminicola } \\
\text { ruminicola }\end{array}$ & 2 & 1 & 3 \\
\hline $\begin{array}{l}\text { Prevotella } \\
\text { heparinolytica }\end{array}$ & 0 & 14 & 14 \\
\hline Prevotella bivia & 0 & 1 & 1 \\
\hline Fusobacterium spp. & 14 & 29 & 43 \\
\hline Bacteroides spp. & 26 & 54 & 80 \\
\hline $\begin{array}{l}\text { Propionebacterium } \\
\text { acnes }\end{array}$ & 1 & 2 & 3 \\
\hline $\begin{array}{l}\text { Cocobacilo, G- com } \\
\text { pigmento negro }\end{array}$ & 3 & 19 & 22 \\
\hline Total & 88 & 291 & 379 \\
\hline
\end{tabular}

RENVERT et al., 1996; FORSBLOM et al., 1997; DOMINGUES et al., 1999). O perfil bioquímico de muitas amostras de Bacteroides não se enquadrou nas tabelas de referência (SNEATH et al.,1986; SUMANNEN et al., 1993; BARON et al., 1994; HOLT et al., 1994; QUINN et al., 1994 KONEMAN et al., 2001), e a utilização de kits comerciais também não permitiu uma completa identificação.

Sobre as espécies bacterianas anaeróbias, Bacteroides pneumosintes, Bacteroides ovatus, Bacteroides eggertii, Bacteroides capilosus, Prevotella ruminicola ruminicola; anaeróbias facultativas/aeróbias: Pasteurella pneumotropica, Pasteurella canes, Pasteurella haemolytica, Lactobacillus salivosus salivosus, Escherichia coli, Klebsiella pneumoniae, Rothia dentocariosa, Arachinia propionica, Moraxella lacunata, Moraxella phenylpyruvica, Micrococcus luteus, Acinetobacter lwofii, Branhamella catarrhalis, Staphylococcus saprophyticus, Staphylococcus hyicus hyicus, Staphylococcus hyicus chromogenes, Staphylococcus xylosus, Staphylococcus saccharolyticus, Staphylococcus auricularis e as leveduras Malassezia pachidermatis e Rhodotorulla spp., não foram encontrados relatos na literatura consultada (NEWMAN et al., 1977; SYED et al., 1981; ISOGAI et al., 1989; HENNET \& HARVEY, 1991; SARKIALA et al., 1993; BOYCE et al., 1995; HARVEY et al., 1995; RENVERT et al., 1996; FORSBLOM et al., 1997; DOMINGUES et al., 1999).

A diferenciação entre certas espécies de Porphyromonas produtoras de colônias com pigmentação negra, freqüentemente isoladas de cães, como $\boldsymbol{P}$. canoris, $\boldsymbol{P}$. cangingivalis, $\boldsymbol{P}$. cansulci, $\boldsymbol{P}$. gingivicanis e P. crevioricanis (COLLINS et al.,1994; HIRASAWA \& TAKADA, 1994; LOVE et al., 1994) e Fusobacterium nucleatum/necrophorum não foi realizada devido a limitações metodológicas.

Tabela 2 - Distribuição de amostras microbianas anaeróbias facultativas isoladas de sítios periodotais saudáveis e com doença periodontal (DP) de 29 cães da raça Pastor Alemão (Minas Gerais, Brasil).

\begin{tabular}{|c|c|c|c|}
\hline \multirow{2}{*}{ Microbiota anaeróbia facultativa } & \multicolumn{2}{|c|}{$\begin{array}{l}\text { Distribuição } \\
\text { das amostras }\end{array}$} & \multirow{2}{*}{ Total } \\
\hline & Saudável & DP & \\
\hline Pasteurella multocida & 1 & 17 & 18 \\
\hline Pasteurella pneumotopica & 1 & 5 & 6 \\
\hline Pasteurella canis & 19 & 20 & 39 \\
\hline Pasteurella haemolytica & 0 & 5 & 5 \\
\hline Lactobacillus salivosus salivosus & 2 & 4 & 6 \\
\hline Escherichia coli & 0 & 12 & 12 \\
\hline Corynebacterium spp. & 9 & 4 & 13 \\
\hline Klebsiella oxyloca & 1 & 0 & 1 \\
\hline Klebsiella pneumoniae & 0 & 1 & 1 \\
\hline Rothia dentocariosa & 4 & 3 & 7 \\
\hline Arachnia propionica & 2 & 7 & 9 \\
\hline Streptococcus $\beta$ hemolítico & 2 & 4 & 6 \\
\hline Enterobacter spp. & 0 & 1 & 1 \\
\hline Staphylococcus epidermides & 6 & 1 & 7 \\
\hline Staphylococcus saprophyticus & 8 & 0 & 8 \\
\hline Staphylococcus waneri & 1 & 0 & 1 \\
\hline Staplycoccus hyicus hyicus & 1 & 0 & 1 \\
\hline Stapphylococcus hyicus chromogenes & 1 & 0 & 1 \\
\hline Staphylococcus xylosus & 4 & 1 & 5 \\
\hline Staphylococcus saccharolyticus & 1 & 1 & 2 \\
\hline Staphylococcus capitis & 1 & 0 & 1 \\
\hline Staphylococcus intermedius & 1 & 1 & 2 \\
\hline Staphylococcus hominis & 5 & 1 & 6 \\
\hline Staphylococcus auriculares & 1 & 0 & 1 \\
\hline Bastonete e cocobacilo G- & 8 & 41 & 49 \\
\hline Cocos $\mathrm{G}+$ & 3 & 4 & 7 \\
\hline Bastonete G+ & 6 & 15 & 21 \\
\hline Total & 88 & 148 & 236 \\
\hline
\end{tabular}

Ciência Rural, v.35, n.2, mar-abr, 2005. 


\begin{tabular}{|c|c|c|c|c|}
\hline Tabela & \multicolumn{4}{|c|}{$\begin{array}{l}\text { Distribuição de amostras microbianas } \\
\text { aeróbias/microaerófilas isoladas de sítios } \\
\text { periodotais saudáveis e com doença } \\
\text { periodontal (DP) de } 29 \text { cães da raça Pastor } \\
\text { Alemão (Minas Gerais, Brasil). }\end{array}$} \\
\hline \multicolumn{2}{|c|}{$\begin{array}{c}\text { Microbiota } \\
\text { aeróbia/microaerófila e } \\
\text { leveduras }\end{array}$} & \multicolumn{2}{|c|}{$\begin{array}{l}\text { Distribuição das } \\
\text { amostras }\end{array}$} & Total \\
\hline & & Saudável & DP & \\
\hline \multicolumn{2}{|c|}{ Moraxella lacunata } & 5 & 8 & 13 \\
\hline \multicolumn{2}{|c|}{ Moraxella phenylpyruvica } & 1 & 1 & 2 \\
\hline \multicolumn{2}{|c|}{ Micrococcus luteos } & 19 & 3 & 22 \\
\hline \multicolumn{2}{|c|}{ Acinetobacter lwofii } & 0 & 2 & 2 \\
\hline \multicolumn{2}{|c|}{ Alcaligenes faecalis } & 1 & 1 & 2 \\
\hline \multicolumn{2}{|c|}{ Branhamella catarrhalis } & 0 & 2 & 2 \\
\hline \multicolumn{2}{|c|}{ Pseudomonas spp. } & 3 & 0 & 3 \\
\hline \multicolumn{2}{|c|}{ Malasezia pachidermatis } & 1 & 9 & 10 \\
\hline \multicolumn{2}{|c|}{ Rhodotorulla spp. } & 0 & 1 & 1 \\
\hline \multicolumn{2}{|l|}{ Total } & 30 & 27 & 57 \\
\hline
\end{tabular}

Segundo os dados mostrados nas Tabelas 1, 2 e 3, a maior parte das espécies encontradas esteve presente tanto nos sítios saudáveis quanto nos lesados, indicando que estas espécies pertencem à microbiota indígena do sulco gengival dos cães, como nos humanos. Quando em equilíbrio, a microbiota atua como barreira natural, protegendo a mucosa oral contra patógenos exógenos (ENGELKIRK et al., 1992). No entanto, alterações locais do equilíbrio ecológico podem resultar em infecções oportunistas (DARVEAU et al., 1997; LANG et al., 1999). Podese observar que a contagem de UFC foi maior nos sítios que apresentavam sinais clínicos de doença periodontal e que o acúmulo de placa tinha uma correlação positiva com o isolamento de anaeróbios. Anaeróbios estritos pertencentes aos gêneros Porphyromonas spp., Fusobacterium spp. e Bacteroides foram prevalentes nos sítios com doença periodontal, onde Pasteurella multocida e Escherichia coli também foram isolados em número considerável. Dados similares foram previamente relatados por SYED et al. (1981) e ISOGAI et al. (1989).

O alto número de Porphyromonas spp. e Fusobacterium spp. encontrado em sítios doentes reforça as evidências da participação destes microrganismos na patogênese da periodontite em cães, como sugerido em outros trabalhos (SYED et al., 1981; ISOGAI et al., 1989; SARKIALA et al., 1993; ALLAKER et al., 1994; BOYCE et al., 1995; RENVERT et al., 1996), similarmente à associação descrita na doença periodontal humana
(SOCRANSKY \& HAFFAJEE, 1999).

A análise estatística mostrou que o isolamento de Porphyromonas spp. catalase positiva, Pasteurella multocida, Escherichia coli e Fusobacterium russi foi maior em sítios com doença periodontal, quando comparado com sítios saudáveis. Por outro lado, o isolamento de Staphylococcus saprophyticus foi superior nos sítios saudáveis dos cães do Grupo II. A contagem de colônias de Fusobacterium nucleatum, Fusobacterium spp. e Bacteroides spp. foi sempre superior em sítios doentes, mas não se observou diferença estatística significativa. Considerando sítios saudáveis, bactérias anaeróbias facultativas foram predominantes, especialmente Staphylococcus saprophyticus. Espécies bacterianas adicionais não mostraram diferenças, em número, em ambos os grupos de cães, considerando material isolado de sítios doentes e saudáveis $(\mathrm{P}>0,05)$.

No presente trabalho, não foi possível a recuperação de amostras de Actinobacillus actinomycetemcomitans. Dados similares foram apresentados por BOYCE et al. (1995) e RENVERT et al. (1996). Segundo PREUS \& OLSEN (1988), não há evidências de que o $\boldsymbol{A}$. actinomycetemcomitans faça parte da microbiota indígena da cavidade oral de cães, dada às dificuldades do seu isolamento. Entretanto, não descartam a possibilidade de os cães terem o $\boldsymbol{A}$. actinomycetemcomitans como microrganismo transiente em sua microbiota, podendo, eventualmente, ser isolado, como mostram BERGLUNDH \& LINDHE (1993). O conhecimento da microbiota oral de cães permite também estabelecer os prováveis patógenos envolvidos nas infecções de feridas por mordidas em animais e em seres humanos, assim como em infecções de feridas cirúrgicas de cães pelo próprio paciente (CARNEIRO et al., 2001) e, consequentemente, orientar na escolha do melhor tratamento. A dificuldade de tratamento das infecções de feridas por mordidas de animais, em seres humanos, foi relatada por FORSBLOM et al. (1997), GOLDSTEIN et al. (1984) e GOLDSTEIN (1998), principalmente devido à falta de conhecimento sobre a composição exata da microbiota indígena da cavidade oral canina, ainda não descrita.

A alta incidência de Pasteurella nos dois grupos de animais estudados, como já relatado por BARON et al. (1994), e a freqüente contaminação de feridas de mordidas de cães em humanos por estes microrganismos (TALAN et al., 1999), sugere que o cão possa ser o reservatório natural desta bactéria. 


\section{CONCLUSÕES}

A microbiota anaeróbia facultativa e aeróbia é predominante nos sítios saudáveis, enquanto a anaeróbia estrita ocorre em maior número nos sítios com gengivite. A microbiota encontrada foi equivalente nos sítios periodontais saudáveis e com gengivite, demonstrando se tratar de microbiota indígena. A boca é colonizada por um incontável número de diferentes organismos, muitos deles já descritos, e há muito o que aprender sobre a patogênese da doença periodontal em cães, no sentido de fornecer suporte clínico, considerando diagnóstico e terapia.

\section{AGRADECIMENTOS}

Este projeto foi financiado pelo CNPq, CAPES, FAPEMIG e PRPq/UFMG. Os autores são gratos a Luzia Rosa Resende da Silva, pela ajuda técnica.

\section{FONTES DE AQUISIÇÃO}

a) Champ Original e Pedigree Champ; b) Rompum $2 \%$ - Bayer S. A. - São Paulo - SP - Brasil; c) Forma Scientifica Company Marietta - OH - USA; d) TSA Difco - Interlab - São Paulo - SP - Brasil; e) Hemina Eqüina Inlab - Interlab - São Paulo - SP Brasil; f) Menadiona Inlab - Interlab - São Paulo - SP - Brasil; g) Yeast Extract Difco - Interlab - São Paulo - SP - Brasil; h) Agar MacConkey - Difco - Interlab - São Paulo - SP - Brasil; i)Agar Sabouraud Difco - Interlab - São Paulo - SP - Brasil; j) Sintomicetina - Medley Ind. Farmacêutica - São Paulo - SP Brasil.

\section{REFERÊNCIAS BIBLIOGRÁFICAS}

ALLAKER, R.P. et al. Prevalence of Eikenella corrodens and Actinobacillus actinomycetemcomitans in the dental plaque of dogs. Veterinary Record, v.134, n.20, p.519$520,1994$.

BARON, E. et al. Diagnostic microbiology. 9.ed. Missouri: Mosby, 1994. 958p.

BERGLUNDH, T.; LINDHE, J. Gingivitis in young and old dogs. Journal of Clinical Periodontology, v.20, p.179-185, 1993.

BOYCE, E. N. et al. Ocurrence of gram-negative black-pigmented anaerobes in subgingival plaque during the development of canine periodontal disease. Clinical Infection Disease, v.20, n.2, p.317319, 1995.

CARNEIRO, L. P. et al. External skeletal fixation in dogs: clinical and microbiological evaluation. Arquivos Brasileiros de Medicina Veterinária e Zootecnia, Belo Horizonte, v.53, n.4, p.437-444, 2001.

COLLINS, M.D. et al. Phylogenetic analysis of members of the genus Porphyromonass and description of Porphyromonas cangingivalis sp. nov. and Porphyromonas cansulci sp. nov.
International Journal of Systematic Bacteriology, v.44, n.4, p.674-679, 1994.

DARVEAU, R.P. et al. The microbial challenge in periodontitis. Periodontology 2000, v.14, p.12-32, 1997.

DOMINGUES, L.M. et al. Microbiota saprófita associada à doença periodontal em cães. Arquivo Brasileiro de Medicina Veterinaria e Zootecnia, v.51, n.4, p.239-232, 1999.

DUARTE, E.R. et al. Prevalence of Malassezia spp. In the ears of asymptomatic cattle and cattle with otitis in Brazil. Medical Mycology, v.37, p.159-162, 1999.

ENGELKIRK, P.G. et al. Principles and practices of clinical anaerobic bacteriology. Belmont : Star, 1992. 462p

FINEGOLD, S.M. Overview of clinically important anaerobes. Clinical Infectious Diseases, v.20, n.2, p.205-207, 1995.

FORSBLOM, B. et al. Characterization of anaerobic, Gramnegative, nonpigmented, saccharolytic rods from subgengival sites in dog. Clinical Infectious Diseases, v.25, n.2, p.100-106, 1997.

GOLDSTEIN, E.J.C. et al. Role of anaerobic bacteria in bitewound infection. Reviews of Infection Diseases, v.6, n.1, p.177183, 1984.

GOLDSTEIN, E.J.C. New horizons in the bacteriology, antimicrobial susceptibility and therapy of animal bite wounds. Journal Medical Microbiology, v.47, p.95-97, 1998.

GÜEHO, E. et al. The genus Malassezia with description of four new species. Antonie Leeuwenhoek, v.9, p.337-355, 1996.

GUILLOT, J. et al. Identification of Malassezia species. A practical approach. Journal Medical Mycology, v.6, n.3, p.103110, 1996

HARVEY, C.E.; EMILY, P.P. Small animal dentistry. USA : Mosby, 1993. 413p.

HARVEY, C.E. et al. Subgengival bactria - comparison of culture results in dogs and cats with gingivitis. Journal Veterinary Dentistry, v.12, n.4, p.147-150, 1995

HENNET, P.R.; HARVEY, C.E. Aerobes in periodontal disease in the dog: a review. Journal Veterinary Dentistry, v.8, n.1, p.9-11, 1991

HIRASAWA, M.; TAKADA, K. Porphyromonas gingivicanis sp. nov. and Porphyromonas crevioricanis sp. nov., isolated from Beagles. International Journal of Systematic Bacteriology, v.44, n.4, p.637-640, 1994

HOLT, J.G. et al. Bergey's manual of determinate bacteriology. 9.ed. Baltimore: Williams \& Wilkins, 1994. 787p.

ISOGAI, E. et al. Oral flora of mongrel and Beagle dogs with periodontal disease. Japanese Journal Veterinary Science, v.51, n.1, p.110-118, 1989.

JOHNSON, R.; BHATTACHARYYA, G. Statistics principles and methods. New York : John Wiley \& Sons, 1986. 578p.

KONEMAN, E.W. et al. Diagnóstico microbiológico, texto e atlas colorido. 5.ed. Rio de Janeiro : MEDSI, 2001. $1465 p$

Ciência Rural, v.35, n.2, mar-abr, 2005. 
LANG, N.P. et al. Placa e cálculos dentais. In: LINDHE, J. Tratado de periodontia clínica e implantologia oral. 3.ed. Rio de Janeiro : Guanabara Koogan, 1999. p.66-91.

LOVE, D.N. et al. Porphyromonas canoris sp. Nov., an asaccharolytic, black-pigmented species from the gengival sulcus of dogs. International Journal of Sistematic Bacteriology, v.44, n.2, p.204-208, 1994

NEWMAN, M.G. et al. The effect of dietary supplements on the flora of periodontal pockets in four Beagle dogs. Journal Periodontology Research, v.12, p.129-134, 1977.

PREUS, H.R.; OLSEN, I. Possible transmittance of $\boldsymbol{A}$. actinomycetemcomitans from a dog to a child with rapidly destructive periodontitis. Journal Periodontology Research, v.23, p.68-71, 1988.

QUINN, P.J. et al. Clinical veterinary microbiology. London: Wolf, 1994. 648p.

RENVERT S. et al. Histologycal and microbiological aspects of ligature-induced periodontitis in Beagle dogs. Journal of Clinical
Periodontology, n.23, p.310-319, 1996.

SARKIALA, E. et al. Clinical, radiological and bacteriological findings in canine periodontitis. Journal of Small Animal Practice, v.34, p.265-270, 1993.

SNEATH, P.H.A. et al. Bergey's manual of systematic bacteriology. Baltimore : Williams \& Wilkins, 1986. V.2, p.965-1599.

SOCRANSKY, S.S.; HAFFAJEE, A.D. Microbiologia da doença periodontal. In: LINDHE, J. Tratado de periodontia clínica e implantologia oral. 3.ed. Rio de Janeiro:Guanabara Koogan, 1999. p.92-126.

SUMMANEN, P. et al. Wadsworth anaerobic bacteriology manual. 5.ed. Belmont:Star, 1993. 230p.

SYED, S.A. et al. The predominant cultivable dental plaque flora of beagle $\operatorname{dog} s$ with periodontitis. Journal of Clinical Periodontology, v.8, p.45-56, 1981.

TALAN, D.A. et al. Bacteriologic analisis of infected dog and cat bites. New England Journal of Medicine, v.340, n.2, p.8592, 1999. 